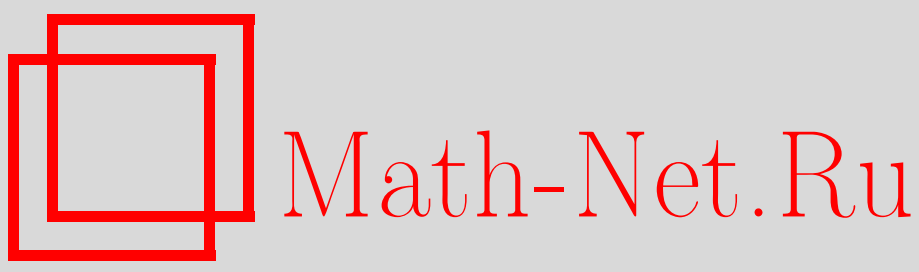

М. О. Авдеева, В. А. Быковский, Аналог теоремы Валена для совместных приближений пары чисел, Матем. сб., 2003, том 194, номер 7, 3-14

DOI: https://doi.org/10.4213/sm749

Использование Общероссийского математического портала Math-Net.Ru подразумевает, что вы прочитали и согласны с пользовательским соглашением http://www . mathnet.ru/rus/agreement

Параметры загрузки:

IP : 35.173 .219 .12

26 апреля 2023 г., 14:53:48 
УДК $511.334+515.178$

М.О. Авдеева, В.А. Быковский

\title{
Аналог теоремы Валена для совместных приближений пары чисел
}

\author{
В работе изучаются свойства совместных диофантовых приближений для двух \\ вещественных чисел. \\ Библиография: 11 названий.
}

\section{Введение}

Пусть $\alpha=\left[t_{0}, t_{1}, \ldots, t_{i}, \ldots\right]$ есть стандартная формальная запись разложения вешественного $\alpha$ в цепную дробь с целой частью $t_{0}=[\alpha]$ и неполными частными $t_{1}, \ldots, t_{i}, \ldots$ (натуральные числа). Для подходящих дробей $a_{i} / q_{i}=\left[t_{0}, t_{1}, \ldots, t_{i}\right] \mathrm{c}$ взаимно простыми целым $a_{i}$ и натуральным $q_{i}$ хорошо известна оценка $\left|\alpha-a_{i} / q_{i}\right|<$ $q_{i}^{-2}$, которую можно представить в виде $\left\|\alpha q_{i}\right\| q_{i}<1$. Здесь $\|x\|$ обозначает расстояние от $x$ до ближайшего целого. К. Вален [1] усилил ее, доказав неравенство

$$
\min \left\{\left\|\alpha q_{i}\right\| q_{i},\left\|\alpha q_{i+1}\right\| q_{i+1}\right\}<\frac{1}{2}
$$

(см. также [2]-[4]). При этом константу $\frac{1}{2}$ из правой части нельзя заменить на меньшее число. По теореме Лагранжа (см. [2]-[4]) знаменатели подходящих дробей $a_{i} / q_{i}$ однозначно определяются условием, что $\left\|\alpha q_{i}\right\|<\left\|\alpha q^{\prime}\right\|$ для всех натуральных $q^{\prime}<q_{i}$. При этом удобно считать, что $q_{0}=1$. Это наблюдение мотивирует следующее

ОПРЕДЕЛЕНИЕ 1. Натуральное $q$ называется подходящим знаменателем $\kappa$ паре вещественных чисел $(\alpha, \beta)$, если для всех натуральных $q^{\prime}<q$ выполняется хотя бы одно из неравенств

$$
\|\alpha q\|<\left\|\alpha q^{\prime}\right\|, \quad\|\beta q\|<\left\|\beta q^{\prime}\right\| .
$$

Очевидно, что любой подходящий знаменатель к $\alpha$ или $\beta$ (в обычном смысле) удовлетворяет определению. Вообще говоря, последовательность подходящих знаменателей к $(\alpha, \beta)$ более "густая" для случайно выбранных $\alpha$ и $\beta$. Она конечна только в случае одновременной рациональности $\alpha$ и $\beta$. Для краткости в дальнейшем прилагательное "подходящий” будем опускать.

Работа вьполнена при финансовой поддержке Российского фонда фундаментальных исследований (грант № 01-01-96301) и Президиума ДВО РАН (грант № 03-1-0-01-003).

$$
\text { (C) М.О. АвдеЕвА, В.А. БЫковский } 2003
$$


ОПРЕДЕЛЕНИЕ 2. Различные знаменатели $q_{1}$ и $q_{2} \mathrm{k}(\alpha, \beta)$ назовем смежжными если не существует отличного от них третьего $q$, для которого

$$
\begin{gathered}
q<\max \left\{q_{1}, q_{2}\right\} \\
\|\alpha q\| \leqslant \max \left\{\left\|\alpha q_{1}\right\|,\left\|\alpha q_{2}\right\|\right\}, \quad\|\beta q\| \leqslant \max \left\{\left\|\beta q_{1}\right\|,\left\|\beta q_{2}\right\|\right\} .
\end{gathered}
$$

Отметим, что в случае $\alpha-\beta \in \mathbb{Z}$ речь идет о знаменателях $q_{i}$ и $q_{i+1}$ для соседних подходящих дробей к $\alpha$. При нецелом $\alpha-\beta$ между смежными $q_{1}$ и $q_{2}$ могут располагаться несколько других знаменателей $q$ к $(\alpha, \beta)$ в каком угодно количестве.

Для любого знаменателя $q$ к $(\alpha, \beta)$ из теоремы Минковского о выпуклом теле немедленно следует неравенство $\|\alpha q\|\|\beta q\| q<1$ (аналог ранее упоминавшейся оценки $\left.\left\|\alpha q_{i}\right\| q_{i}<1\right)$. В работе [5] для смежных $q_{1}$ и $q_{2} \mathrm{k}(\alpha, \beta)$ было доказано неравенство

$$
\min \left\{\left\|\alpha q_{1}\right\|\left\|\beta q_{1}\right\| q_{1},\left\|\alpha q_{2}\right\|\left\|\beta q_{2}\right\| q_{2}\right\}<\frac{1}{2}
$$

(аналог оценки Валена из (0.1)). При этом постоянную $\frac{1}{2}$ нельзя заменить на меньшую.

Наша цель состоит в том, чтобы усилить (0.2). Для этого сформулируем

ОПРЕДЕЛЕНИЕ 3 . Назовем три знаменателя $q_{1}, q_{2}, q_{3}$ к $(\alpha, \beta)$ смежныьми, если они попарно смежны и не сушествует четвертого $q$ (в том числе совпадаюшего $\mathrm{c}$ одним из $\left.q_{1}, q_{2}, q_{3}\right)$, для которого

$$
\begin{gathered}
q<\max \left\{q_{1}, q_{2}, q_{3}\right\}, \\
\|\alpha q\|<\max _{i=1,2,3}\left\{\left\|\alpha q_{i}\right\|\right\}, \quad\|\beta q\|<\max _{i=1,2,3}\left\{\left\|\beta q_{i}\right\|\right\} .
\end{gathered}
$$

Главным результатом работы является

ТЕОрема. Пусть $q_{1}, q_{2}, q_{3}$ суть смежние знаменатели $\kappa(\alpha, \beta)$, ни один из которых не равен сумме двух других. Тогда

$$
\min _{i=1,2,3}\left\{\left\|\alpha q_{i}\right\|\left\|\beta q_{i}\right\| q_{i}\right\}<\frac{1}{3} .
$$

Постоянную $\frac{1}{3}$ в правой части нельзя заменить на меньшую. Следует отметить, что для смежных троек с $q_{1}<q_{2}<q_{3}$ условие $q_{1}+q_{2}=q_{3}$ выполняется для почти всех $(\alpha, \beta)$ (по мере Лебега) с хотя и малой, но положительной частотой. Этот вывод основан на эвристических соображениях, на которых мы не будем здесь останавливаться.

\section{§1. Свойства смежных знаменателей}

В этом параграфе изучаются особенности взаимного расположения смежных знаменателей, которые нам понадобятся при доказательстве теоремы. В идейном плане мы опираемся на основополагающие работы $Г$. $\Phi$. Вороного [6] и Г. Минковского [7]. Речь идет о теориях смежных минимумов (Вороной) и минимальных базисах (Минковский) для трехмерных полных решеток. Мы также используем некоторые технические приемы из работ [8] и [9]. 
Пару $(\alpha, \beta)$, начиная с этого момента, мы будем считать фиксированной. Положим по определению $((x))=\left\{x+\frac{1}{2}\right\}-\frac{1}{2} \in\left[-\frac{1}{2}, \frac{1}{2}\right)$ для любого вешественного $x$. При этом $\|x\|=|((x))|$. Пусть

$$
D\left(q_{1}, q_{2}, q_{3}\right)=\operatorname{det}\left(\begin{array}{ccc}
q_{1} & q_{2} & q_{3} \\
\left(\left(\alpha q_{1}\right)\right) & \left(\left(\alpha q_{2}\right)\right) & \left(\left(\alpha q_{3}\right)\right) \\
\left(\left(\beta q_{1}\right)\right) & \left(\left(\beta q_{2}\right)\right) & \left(\left(\beta q_{3}\right)\right)
\end{array}\right) .
$$

Лемма 1. Если $q_{1}, q_{2}, q_{3}$ составляют тройку смежных знаменателей $\kappa$ $(\alpha, \beta)$, то $D\left(q_{1}, q_{2}, q_{3}\right)=0, \pm 1$. При әтом определитель равен нулю только в случае, когда один из столбцов матрицы в (1.1) равен сумме двух других.

ДоКАЗАТЕЛЬСтво. Определим полную трехмерную решетку

$\Gamma(\alpha, \beta)=\{(l, \alpha l+m, \beta l+n)=l(1, \alpha, \beta)+m(0,1,0)+n(0,0,1): l, m, n \in \mathbb{Z}\}$,

целочисленно порожденную базисом $(1, \alpha, \beta),(0,1,0),(0,0,1)$. Ее определитель равен 1. Векторы

$$
Q_{i}=\left(q_{i},\left(\left(\alpha q_{i}\right)\right),\left(\left(\beta q_{i}\right)\right)\right), \quad i=1,2,3
$$

будут узлами $\Gamma(\alpha, \beta)$. Они целочисленно выражаются через базис. Поэтому $D\left(q_{1}, q_{2}, q_{3}\right)$ - целое число.

Рассмотрим сначала случай $D\left(q_{1}, q_{2}, q_{3}\right)=0$. Это означает, что при некоторых целых $a_{1}, a_{2}, a_{3}$ одновременно выполняется равенство

$$
a_{1} Q_{1}+a_{2} Q_{2}+a_{3} Q_{3}=(0,0,0) .
$$

Ввиду симметрии без ограничения обшности можно считать, что $\left|a_{1}\right| \geqslant\left|a_{2}\right| \geqslant\left|a_{3}\right|$. Разделив обе части (1.2) на $a_{1}$, получим еше одно равенство

$$
Q_{1}+\theta_{2} Q_{2}+\theta_{3} Q_{3}=(0,0,0) .
$$

Выберем $\varepsilon_{2}, \varepsilon_{3} \in\{0, \pm 1\}$ из условий

$$
\theta_{2}=\varepsilon_{2}-\theta_{2}^{\prime}, \quad\left|\theta_{2}^{\prime}\right| \leqslant \frac{1}{2}, \quad \theta_{3}=\varepsilon_{3}-\theta_{3}^{\prime}, \quad\left|\theta_{3}^{\prime}\right| \leqslant \frac{1}{2} .
$$

Тогда

$$
Q=Q_{1}+\varepsilon_{2} Q_{2}+\varepsilon_{3} Q_{3}=\theta_{2}^{\prime} Q_{2}+\theta_{3}^{\prime} Q_{3} .
$$

Поскольку $Q$ - узел $\Gamma(\alpha, \beta)$, то

$$
Q=(l, \alpha l+m, \beta l+n)
$$

для некоторых целых $l, m, n$. Так как $\left|\theta_{2}^{\prime}\right| \leqslant \frac{1}{2}$ и $\left|\theta_{3}^{\prime}\right| \leqslant \frac{1}{2}$, то

$$
\begin{aligned}
\left|\theta_{2}^{\prime} q_{2}+\theta_{3}^{\prime} q_{3}\right| & \leqslant \frac{1}{2} q_{2}+\frac{1}{2} q_{3}, \\
\left|\theta_{2}^{\prime}\left(\left(\alpha q_{2}\right)\right)+\theta_{3}^{\prime}\left(\left(\alpha q_{3}\right)\right)\right| & \leqslant \frac{1}{2}\left\|\alpha q_{2}\right\|+\frac{1}{2}\left\|\alpha q_{3}\right\|, \\
\left|\theta_{2}^{\prime}\left(\left(\beta q_{2}\right)\right)+\theta_{3}^{\prime}\left(\left(\beta q_{3}\right)\right)\right| & \leqslant \frac{1}{2}\left\|\beta q_{2}\right\|+\frac{1}{2}\left\|\beta q_{3}\right\| .
\end{aligned}
$$


Предположим, что $l \neq 0$. Тогда для $q=|l|$ выполняются неравенства

$$
\begin{gathered}
q<\max \left\{q_{2}, q_{3}\right\} \\
\|\alpha q\| \leqslant \max \left\{\left\|\alpha q_{2}\right\|,\left\|\alpha q_{3}\right\|\right\}, \quad\|\beta q\| \leqslant \max \left\{\left\|\beta q_{2}\right\|,\left\|\beta q_{3}\right\|\right\} .
\end{gathered}
$$

Поскольку $q_{2}$ и $q_{3}$ смежные, то $q$ совпадает с одним из них (см. определение 2). При этом мы воспользовались следуюшим очевидньм фактом. Для любого натурального $q$ найдется знаменатель $q^{\prime}$ к $(\alpha, \beta)$, для которого

$$
q^{\prime} \leqslant q, \quad\left\|\alpha q^{\prime}\right\| \leqslant\|\alpha q\|, \quad\left\|\beta q^{\prime}\right\| \leqslant\|\beta q\| .
$$

Таким образом, имеет место соотношение $Q_{1}=\tau_{2} Q_{2}+\tau_{3} Q_{3}$ с $\tau_{2}, \tau_{3} \in\{0, \pm 1, \pm 2\}$ и $\left|\tau_{2}\right|+\left|\tau_{3}\right| \leqslant 3$. Если $\tau_{2}= \pm 2$, то $Q_{2}=\left(1 / \tau_{2}\right) Q_{1}-\left(\tau_{3} / \tau_{2}\right) Q_{3}$ нарушает смежность $q_{1}$ и $q_{3}$. Следовательно, $\tau_{2} \neq \pm 2$. По этой же причине $\tau_{3} \neq \pm 2$. Поскольку $q_{1}, q_{2}, q_{3}$ попарно различны, то $\tau_{2} \neq 0$ и $\tau_{3} \neq 0$. Поэтому $\tau_{2}, \tau_{3} \in\{ \pm 1\}$. Таким образом, в рассматриваемом случае один из столбцов матрицы (1.1) равен сумме двух других. В частности, одно из чисел $q_{1}, q_{2}, q_{3}$ есть сумма остальных двух. Из теоремы 2 , доказанной в работе [9], непосредственно следует, что в невырожденном случае $D\left(q_{1}, q_{2}, q_{3}\right)= \pm 1$. Лемма 1 полностью доказана.

\section{§2. Минимальные и приведенные матрицы}

Рассмотрим множество всех матриц

$$
M=\left(\begin{array}{lll}
x_{11} & x_{12} & x_{13} \\
x_{21} & x_{22} & x_{23} \\
x_{31} & x_{32} & x_{33}
\end{array}\right)
$$

с ненулевьми $x_{i j}$ и $D(M)=\operatorname{det}(M) \neq 0$.

ОПРЕДЕЛЕНИЕ 4. Назовем минимальными те матрицы $M$, у которых

1) для некоторой перестановки $(1,2,3) \mapsto(i, j, k)$ элементы $x_{1 i}, x_{2 j}, x_{3 k}$ максимальны по абсолютной величине в соответствуюших им строках;

2) если в какой-то строке все элементы по абсолютной величине равны, то это свойство вьполняется и для остальных двух;

3) для любых $\varepsilon_{1}, \varepsilon_{2}, \varepsilon_{3} \in\{0, \pm 1\}$, никакие два из которых одновременно не равны нулю, найдется номер $i$ такой, что

$$
\left|\varepsilon_{1} x_{i 1}+\varepsilon_{2} x_{i 2}+\varepsilon_{3} x_{i 3}\right| \geqslant \max \left\{\left|x_{i 1}\right|,\left|x_{i 2}\right|,\left|x_{i 3}\right|\right\} .
$$

Рассмотрим матрицы вида

$$
\left(\begin{array}{ccc}
x_{1} & -y_{1} & z_{1} \\
x_{2} & y_{2} & -z_{2} \\
x_{3} & y_{3} & z_{3}
\end{array}\right), \quad\left(\begin{array}{ccc}
x_{1} & -y_{1} & -z_{1} \\
x_{2} & y_{2} & -z_{2} \\
x_{3} & y_{3} & z_{3}
\end{array}\right)
$$

с положительньми $x_{i}, y_{i}, z_{i}$, у которых

I) $\max \left\{x_{1}, z_{1}\right\} \leqslant y_{1}, \max \left\{y_{2}, z_{2}\right\} \leqslant x_{2}, \max \left\{x_{3}, y_{3}\right\} \leqslant z_{3}$;

II) для первого вида выполняется хотя бы одно из неравенств $z_{1} \leqslant x_{1}, y_{2} \leqslant z_{2}$;

III) для второго вида $y_{3} \leqslant x_{3}$ и $x_{2} \leqslant y_{2}+z_{2}$;

IV) имеет место условие 2) из определения 4. 
ЛЕмма 2. Любая матрица вида (2.1), удовлетворяющая условиям I)-IV), минимальна.

ДоказАтельство. Рассмотрим матрицы первого вида из (2.1). Сначала заметим, что

$$
\operatorname{det}(M)=x_{1} y_{2} z_{3}+x_{3} y_{1} z_{2}+x_{2} y_{3} z_{1}-x_{3} y_{2} z_{1}+x_{2} y_{1} z_{3}+x_{1} y_{3} z_{2}>0
$$

поскольку $x_{2} y_{1} z_{3} \geqslant x_{3} y_{2} z_{1}$ в соответствии с I). Это же условие обеспечивает выполнимость 1), а также 3) в случае, когда одно из чисел $\varepsilon_{1}, \varepsilon_{2}, \varepsilon_{3}$ равно нулю. Рутинная очевидная проверка показьвает, что при дополнительном условии II) выполняется 3) во всех остальных случаях с $\varepsilon_{i} \neq 0, i=1,2,3$. Для матриц второго вида из (2.1)

$$
\operatorname{det}(M)=x_{1} y_{2} z_{3}+x_{3} y_{1} z_{2}-x_{2} y_{3} z_{1}+x_{3} y_{2} z_{1}+x_{2} y_{1} z_{3}+x_{1} y_{3} z_{2}>0
$$

так как $x_{2} y_{1} z_{3} \geqslant x_{2} y_{3} z_{1}$ в соответствии с условием I). Для выполнимости 1) также достаточно I). Если $\varepsilon_{1} \neq 0$, то 3 ) следует из I) и неравенства $y_{3} \leqslant x_{3}$ из III). Такая же картина и для $\left(\varepsilon_{1}, \varepsilon_{2}, \varepsilon_{3}\right)= \pm(0,1,1)$. Неравенство $x_{2} \leqslant y_{2}+z_{2}$ из III) необходимо лишь в случае $\left(\varepsilon_{1}, \varepsilon_{2}, \varepsilon_{3}\right)= \pm(0,1,-1)$. Лемма 2 полностью доказана.

Назовем матрицы $M$ и $M^{\prime}$ әквивалентными, если одна получается из другой с помощью некоторой последовательности преобразований вида

а) перестановка строк или столбцов;

б) изменение знака у строки или столбца.

Легко видеть, что если матрица минимальна, то и эквивалентная ей такая же.

ЛЕмма 3. Любая минимальная матрица $M$ әквивалентна одной из матрии (2.1), удовлетворяющей условиям I)-IV).

ДоКАЗАТЕльство. Из условия 1) определения 4 немедленно следует, что $M$ эквивалентна матрице вида

$$
\left(\begin{array}{ccc}
x_{11} & -y_{1} & x_{13} \\
x_{2} & x_{21} & x_{23} \\
x_{31} & x_{32} & z_{3}
\end{array}\right)
$$

где $x_{2}, y_{1}, z_{3}$ положительны и максимальны в строках по абсолютной величине. Если элемент $x_{11}$ отрицателен, то переменой знаков в первой строке и втором столбце сделаем его положительным. Также поменяем знаки в третьей строке и третьем столбце, если элемент $x_{31}$ отрицателен. В результате получим матрицу вида

$$
\left(\begin{array}{ccc}
x_{1} & -y_{1} & x_{13} \\
x_{2} & x_{21} & x_{23} \\
x_{3} & x_{32} & z_{3}
\end{array}\right)
$$

с положительньми $x_{1}, x_{2}, x_{3}, y_{1}, z_{3}$.

Предположим, что $x_{21}<0$. Из условия 3$)$ определения 4 с $\left(\varepsilon_{1}, \varepsilon_{2}, \varepsilon_{3}\right)=(1,1,0)$ следует, что $x_{32}>0$. По этой же причине $x_{13}$ и $x_{23}$ имеют разные знаки. Рассмотрим случай, когда $x_{13}>0$. Тогда $x_{23}<0$. Переставим вторую и третью строки, а затем первый и третий столбцы. В результате получим матрицу

$$
\left(\begin{array}{ccc}
x_{13} & -y_{1} & x_{1} \\
z_{3} & x_{32} & x_{3} \\
x_{23} & x_{21} & x_{2}
\end{array}\right) .
$$


Поменяв знак в третьей строке, а потом в третьем столбце, получим матрицу второго вида из (2.1). Пусть теперь в $(2.2) x_{13}<0$. Тогда $x_{23}>0$. Рассмотрим вектор

$$
\left(x_{1}-y_{1}-x_{13}, x_{2}+x_{21}-x_{23}, x_{3}+x_{32}-z_{3}\right) .
$$

Мы сложили первые два столбца из (2.2) и отняли третий. Из максимальности $x_{2}, y_{1}, z_{3}$ следует, что

$$
\left|x_{1}-y_{1}-x_{13}\right| \leqslant y_{1}, \quad\left|x_{2}+x_{21}-x_{23}\right| \leqslant x_{2}, \quad\left|x_{3}+x_{32}-z_{3}\right| \leqslant z_{3} .
$$

Так как матрица минимальная, то хотя бы одно из этих неравенств должно быть равенством. Но это возможно только в случае, если все элементы соответствующей строки равны по абсолютной величине. А тогда в соответствии с 2) определения 4 это выполнено и для остальных двух строк. Такая матрица эквивалентна $M$ из (2.1) как первого, так и второго вида.

Предположим, что в (2.2) $x_{21}>0$. Рассмотрим случай $x_{32}>0$. Элемент $x_{23}$ не может быть положительным по причине условия 3$)$ определения $4 \mathrm{c}\left(\varepsilon_{1}, \varepsilon_{2}, \varepsilon_{3}\right)=$ $(0,1,-1),(1,0,-1)$. Значит, $x_{23}<0$ и мы приходим к одному из двух случаев в (2.1). Пусть теперь $x_{32}<0$. Из условия 3$)$ определения 4 следует, что $x_{13}<0$ и $x_{23}>0$. То есть речь идет о матрище вида

$$
\left(\begin{array}{ccc}
x_{1} & -y_{1} & x_{13} \\
x_{2} & x_{21} & x_{23} \\
x_{3} & -x_{32} & z_{3}
\end{array}\right)
$$

с положительными $x_{i j}$. Переставив первую и вторую строки, а затем первый и второй столбцы, получим матрицу

$$
\left(\begin{array}{ccc}
x_{21} & x_{2} & x_{23} \\
-y_{1} & x_{1} & -x_{13} \\
-x_{32} & x_{3} & z_{3}
\end{array}\right) .
$$

Меняя последовательно знаки во второй и третьей строках, а затем во втором и третьем столбцах, получим матрицу второго вида из (2.1). Таким образом, все случаи разобраны и лемма 3 полностью доказана.

ОПРЕДЕЛЕНИЕ 5. Назовем любую матрицу $M$ из (2.1), удовлетворяющую условиям I)-III) (условие IV) опускается), приведенной.

\section{§3. Доказательство основного результата}

Заметим, что утверждение сформулированной во введении теоремы тривиально, если хотя бы одно из чисел $\left\|\alpha q_{i}\right\|,\left\|\beta q_{i}\right\|, i=1,2,3$, равно нулю. Поэтому мы будем предполагать, что элементы невырожденной матрицы из (1.1), определитель которой есть $D(\ldots)$, отличны от нуля. При этом она попадает в разряд приведенных, если $q_{1}, q_{2}, q_{3}$ составляют тройку смежных знаменателей к $(\alpha, \beta)$. Последнее непосредственно следует из условий в определении 3 , поскольку целочисленная комбинация $\varepsilon_{1} Q_{1}+\varepsilon_{2} Q_{2}+\varepsilon_{3} Q_{3}$ есть узел решетки с базисом $Q_{1}, Q_{2}, Q_{3}$. 
ЗАмечАниЕ 1 . Так как $q_{1}, q_{2}, q_{3}$ попарно различны, то в рассматриваемом случае равенство $\left\|\alpha q_{1}\right\|=\left\|\alpha q_{2}\right\|=\left\|\alpha q_{3}\right\|$ не может выполняться (см. условие 2) из определения 4). То же самое верно и при замене $\alpha$ на $\beta$.

По изложенным причинам главный результат непосредственно следует из оценки

$$
\min \left\{x_{1} x_{2} x_{3}, y_{1} y_{2} y_{3}, z_{1} z_{2} z_{3}\right\}<\frac{1}{3} D(M)
$$

для минимальных матриц вида (2.1), у которых ни одна строка не состоит из одинаковых по абсолютной величине чисел.

Положим

$$
\begin{gathered}
X=x_{1} x_{2} x_{3}, \quad Y=y_{1} y_{2} y_{3}, \quad Z=z_{1} z_{2} z_{3}, \\
R=\min \{X, Y, Z\} .
\end{gathered}
$$

В дальнейшем при акцентировании внимания на каком либо из элементов матрицы $M$ мы будем использовать следуюшие обозначения:

$$
\begin{gathered}
X=X\left(x_{1}\right)=X\left(x_{2}\right)=X\left(x_{3}\right), \quad Y=Y\left(y_{1}\right)=Y\left(y_{2}\right)=Y\left(y_{3}\right), \\
D(M)=D\left(x_{1}\right)=\cdots=D\left(z_{3}\right), \quad R=R\left(x_{1}\right)=\cdots=R\left(z_{3}\right) .
\end{gathered}
$$

ЛЕмма 4. Пусть $M$ - приведенная матрица первого вида из (2.1), для которой $z_{1} \leqslant x_{1}$ u X=R. Тогда

$$
R \leqslant \frac{1}{4} D(M)
$$

ДокАЗАТЕЛьство. Применяя неравенство Коши к пяти положительньм слагаемым в разложении $D(M)$ по произведениям троек элементов $M$, получим

$$
\begin{aligned}
D(M) & \geqslant 5\left(x_{1} y_{2} z_{3} \cdot x_{3} y_{1} z_{2} \cdot x_{2} y_{3} z_{1} \cdot x_{2} y_{1} z_{3} \cdot x_{1} y_{3} z_{2}\right)^{1 / 5}-x_{3} y_{2} z_{1} \\
& =5(X Y Z)^{2 / 5}\left(x_{3} y_{2} z_{1}\right)^{-1 / 5}-x_{3} y_{2} z_{1} .
\end{aligned}
$$

Так как $x_{3} y_{2} z_{1} \leqslant x_{3} x_{2} x_{1}=X$, то

$$
D(M) \geqslant 5(X Y Z)^{2 / 5} X^{-1 / 5}-X \geqslant 5 X^{6 / 5} X^{-1 / 5}-X=4 X .
$$

Лемма 4 доказана.

Точно так же доказьвается

Лемма 5. Пусть $M-$ приведенная матрица первого вида из (2.1), для которой $y_{2} \leqslant z_{2}$ u $Z=R$. Тогда

$$
Z \leqslant \frac{1}{4} D(M)
$$

ЛЕмма 6. Пусть $M$ - приведенная матрица первого вида из (2.1), для которой $z_{1} \leqslant x_{1}$. Тогда

$$
R \leqslant \frac{1}{4} D(M)
$$


ДокАЗАТЕЛЬСТво. Раскладывая определитель $M$ по второму столбцу, получаем

$$
D(M)=y_{1}\left(x_{2} z_{3}+x_{3} z_{2}\right)+y_{2}\left(x_{1} z_{3}-x_{3} z_{1}\right)+y_{3}\left(x_{1} z_{2}+x_{2} z_{1}\right) .
$$

Все три слагаемых неотрицательны, поскольку $x_{1} \geqslant z_{1}$ и $z_{3} \geqslant x_{3}$. Зафиксируем все элементы $M$, за исключением $y_{2}$. Предположим, что $Y\left(y_{2}\right)>R\left(y_{2}\right)$. При уменьшении положительного $y_{2}$ свойство приведенности $M$ не нарушается. Поэтому

$$
\frac{R\left(y_{2}\right)}{D\left(y_{2}\right)} \leqslant \frac{R\left(y_{2}^{\prime}\right)}{D\left(y_{2}^{\prime}\right)},
$$

где $y_{2}^{\prime}$ есть наибольшее $y \in\left(0, y_{2}\right)$, для которого $Y(y)=R(y)$. Следовательно, мы можем считать, что $Y=R$. При разложении определителя $M$ по первому столбцу получим

$$
D(M)=x_{1}\left(y_{2} z_{3}+y_{3} z_{2}\right)+x_{2}\left(y_{3} z_{1}+y_{1} z_{3}\right)+x_{3}\left(y_{1} z_{2}-y_{2} z_{1}\right) .
$$

Зафиксируем все элементы $M$, за исключением $x_{3}$. Предположим, что $X\left(x_{3}\right)>Y$.

Рассмотрим случай $y_{1} z_{2}-y_{2} z_{1}>0$. При уменњшении $x_{3}$ приведенность $M$ не нарушается. Поэтому

$$
\frac{R\left(x_{3}\right)}{D\left(x_{3}\right)}<\frac{R\left(x_{3}^{\prime}\right)}{D\left(x_{3}^{\prime}\right)}
$$

где $x_{3}^{\prime}$ есть наибольшее $x \in\left(0, x_{3}\right)$, для которого $X(x)=Y$. Поэтому мы можем считать, что $X=R$ и воспользоваться леммой 4 .

Пусть теперь $y_{1} z_{2}-y_{2} z_{1} \leqslant 0$. Величина

$$
\frac{R\left(x_{3}\right)}{D\left(x_{3}\right)}
$$

при возрастании $x_{3}$ не убывает и поэтому ее достаточно оценить при $x_{3}=z_{3}$. Через $x_{1}^{\prime}$ обозначим наименшшее $x_{1}$, удовлетворяюшее условию $x_{1} \geqslant z_{1}$, для которого $X\left(x_{1}^{\prime}\right) \geqslant Y$. Если $x_{1}^{\prime}>z_{1}$, то

$$
\frac{R\left(x_{1}\right)}{D\left(x_{1}\right)} \leqslant \frac{R\left(x_{1}^{\prime}\right)}{D\left(x_{1}^{\prime}\right)}=\frac{X\left(x_{1}^{\prime}\right)}{D\left(x_{1}^{\prime}\right)}
$$

и нужная оценка следует из леммы 4 . Предположим теперь, что $x_{1}^{\prime}=z_{1}$. Таким образом, неравенство леммы осталось доказать только для приведенных матриц вида

$$
\left(\begin{array}{ccc}
x & -y_{1} & x \\
x_{2} & y_{2} & -z_{2} \\
z & y_{3} & z
\end{array}\right)
$$

с положительньми $x, x_{2}, y_{1}, y_{2}, y_{3}, z, z_{2}$, для которых $x \leqslant y_{1}, \max \left\{y_{2}, z_{2}\right\} \leqslant x_{2}$, $y_{3} \leqslant z$. В этом случае по неравенству Коши

$$
D(M)=z y_{1} z_{2}+x x_{2} y_{3}+z x_{2} y_{1}+x y_{3} z_{2} \geqslant 4\left(x x_{2} y_{1} y_{3} z z_{2}\right)^{1 / 2} .
$$

При этом $x z z_{2}=Z, x_{2} y_{1} y_{3} \geqslant y_{1} y_{2} y_{3}=Y$. Следовательно,

$$
D(M) \geqslant 4 \sqrt{Y Z} \geqslant 4 \min \{Y, Z\} \geqslant 4 \min \{X, Y, Z\} .
$$

Лемма 6 полностью доказана. 
Лемма 7. Пусть $M$ - приведенная матрица первого вида из (2.1), для которой $y_{2} \leqslant z_{2}$. Тогда

$$
R \leqslant \frac{1}{4} D(M)
$$

ДокАЗАТЕльство. Оно проводится точно так же, как для предыдущей леммы. При этом вместо леммы 4 используется лемма 5. На первоначальном этапе определитель раскладывается по первому столбцу (приведено в доказательстве леммы 6). За счет уменьшения $x_{3}$ добиваемся выполнения равенства $X=R$. Затем вьписываем разложение

$$
D(M)=z_{1}\left(x_{2} y_{3}-x_{3} y_{2}\right)+z_{2}\left(x_{1} y_{3}+x_{3} y_{1}\right)+z_{3}\left(x_{1} y_{2}+x_{2} y_{1}\right)
$$

по третьему столбцу. При $x_{2} y_{3}-x_{3} y_{2}>0$ за счет уменњшения $z_{1}$ приходим к случаю $Z=R$ и применяем лемму 5 . В случае $x_{2} y_{3}-x_{3} y_{2} \leqslant 0$ увеличиваем $z_{1}$ до $y_{1}$, а потом с помощью леммы 5 переходим к $z_{2}=y_{2}$. В конце используется по той же схеме неравенство Коши для четырех слагаемых. Лемма доказана.

ЗАмЕчАниЕ 2. Для приведенных матриц первого вида из (2.1) мы получили даже более сильное нестрогое неравенство с постоянной $\frac{1}{4}$ вместо $\frac{1}{3}$ в (3.1). Из хода рассуждений видно, что оно превращается в равенство только при $x_{i}=y_{i}=z_{i}$, $i=1,2,3$.

ЛЕмма 8. Пусть $M$ - приведенная матрица второго вида из (2.1), для которой $x_{1} \leqslant z_{1}$. Тогда

$$
R \leqslant \frac{1}{3} D(M)
$$

ДоКАЗАТЕЛЬСтво. В разложении по второму столбцу

$$
D(M)=y_{1}\left(x_{3} z_{2}+x_{2} z_{3}\right)+y_{2}\left(x_{1} z_{3}+x_{3} z_{1}\right)+y_{3}\left(x_{1} z_{2}-x_{2} z_{1}\right)
$$

$x_{1} z_{2}-x_{2} z_{1} \leqslant z_{1}\left(z_{2}-x_{2}\right) \leqslant 0$. Поэтому отношение $R\left(y_{3}\right) / D\left(y_{3}\right)$ не уменьшится, если заменить $y_{3}$ на $x_{3}$. При этом приведенность $M$ не нарушается. По той же причине, принимая во внимание разложение

$$
D(M)=z_{1}\left(x_{3} y_{2}-x_{2} y_{3}\right)+z_{2}\left(x_{3} y_{1}+x_{1} y_{3}\right)+z_{3}\left(x_{1} y_{2}+x_{2} y_{1}\right)
$$

по третьему столбцу с $x_{3}=y_{3}$, мы можем считать, что $y_{1}=z_{1}$. Так как доказываемое неравенство однородно по строкам, то положим $x_{1}=x_{2}=x_{3}=1, y_{1}=z_{1}=x$, $y_{2}=u, z_{2}=v, y_{3}=1, z_{3}=y$. При этом

$$
1 \leqslant x, \quad 1 \leqslant y, \quad 0<u, v \leqslant 1, \quad 1 \leqslant u+v .
$$

Таким образом, при рассматриваемых значениях параметров достаточно доказать неравенство

$$
\gamma(x, y, u, v)=\frac{\min \{1, x u, x v y\}}{x y+x u+x v+u y+v-x} \leqslant \frac{1}{3} .
$$

Пусть $u \leqslant v$. Тогда $\min \{1, x u, x v y\}=\min \{1, x u\}$. Поэтому

$$
\gamma(x, y, u, v) \leqslant \gamma(x, 1, u, v)=\frac{\min \{1, x u\}}{(x+1)(u+v)} .
$$


Так как

$$
(x+1)(u+v)=x u+x v+(u+v) \geqslant 2 x u+1,
$$

то

$$
\gamma(x, y, u, v) \leqslant \frac{\min \{1, x u\}}{2 x u+1} \leqslant \frac{1}{3} .
$$

Пусть $u>v$. На промежутке $[u / v, \infty)$ функция $\gamma(x, y, u, v)$ убывает по $y$. Поэтому для $y \in[u / v, \infty)$ выполняется неравенство

$$
\gamma(x, y, u, v) \leqslant \gamma\left(x, \frac{u}{v}, u, v\right)=\frac{\min \{1, x u\}}{x u / v+x u+x v+u^{2} / v+v-x} .
$$

Последняя дробь максимальна по $x$ при $x=1 / u$. Следовательно, в рассматриваемом случае

$$
\gamma(x, y, u, v) \leqslant \frac{1}{1 / v+1+v / u+u^{2} / v+v-1 / u} .
$$

Осталось доказать неравенство

$$
\frac{1}{v}+\frac{v}{u}+\frac{u^{2}}{v}+v-\frac{1}{u} \geqslant 2
$$

которое эквивалентно неравенству

$$
(1+u) v^{2}-(2 u+1) v+u^{3}+u \geqslant 0 .
$$

Для фиксированного $u \in\left[\frac{1}{2}, 1\right]$ его левая часть как функция от $v$ имеет минимум в точке $v_{0}=(2 u+1) /(2 u+2)$. Последняя лежит левее $u$ только для $1 / \sqrt{2}<u \leqslant 1$. Но в этом случае

$$
\frac{1}{v}+\frac{v}{u}+\frac{u^{2}}{v}+v-\frac{1}{u}>\frac{u^{2}}{v}+\frac{v}{u}+v \geqslant \frac{1}{2 v}+2 v \geqslant 2 .
$$

При этом мы воспользовались неравенством $u>v$. Осталось рассмотреть случай $1 / 2 \leqslant u \leqslant 1 / \sqrt{2}$. Для него нужный минимум по $v$ достигается в точке $v=u$. При этом

$$
(1+u) u^{2}-(2 u+1) u+u^{3}+u=2 u^{3}-u^{2}=u^{2}(2 u-1) \geqslant 0 .
$$

Неравенство $\gamma(x, y, u, v) \leqslant \frac{1}{3}$ доказано для $y \geqslant u / v$. Пусть теперь $1 \leqslant y<u / v$. Тогда

$$
\gamma(x, y, u, v)=\frac{\min \{1, x v y\}}{x y+x u+x v+u y+v-x} .
$$

Как функция от $y$ эта величина принимает максимальное значение при $y=1$, если $x v \geqslant 1$, и при $y=1 /(x v)$, если $x v<1$. В первом случае остаются в силе выкладки для ранее рассмотренного варианта $u \leqslant v$. Пусть теперь $x v<1$. Тогда

$$
\gamma(x, y, u, v) \leqslant \gamma\left(x, \frac{1}{x v}, u, v\right)=\frac{1}{1 / v+x u+x v+u /(x v)+v-x} .
$$

Поскольку последнее выражение убывает по $x$, то при $u>v$

$$
\gamma(x, y, u, v) \leqslant \frac{1}{1 / v+u / v+u+2 v-1} \leqslant \frac{1}{1 / v+3 v} \leqslant \frac{1}{2 \sqrt{3}}=\frac{\sqrt{3}}{2} \cdot \frac{1}{3}<\frac{1}{3} .
$$

Лемма 8 полностью доказана. 
Лемма 9. Пусть $M$ - приведенная матрица $M$ второго вида из (2.1), для которой $x_{1} \geqslant z_{1}, x_{3}=y_{3}$. Тогда

$$
R \leqslant \frac{1}{3} D(M)
$$

ДокАЗАТЕльство. Заметим, что коэффициент $x_{3} y_{2}-x_{2} y_{3}\left(x_{3}=y_{3}\right.$, а также $y_{2} \leqslant x_{2}$ в соответствии с условием I) при $z_{1}$ в разложении $D(M)$ по третьему столбцу не превосходит нуля. Поэтому $R\left(z_{1}\right) / D\left(z_{1}\right) \leqslant R\left(x_{1}\right) / D\left(x_{1}\right)$ с условием $x_{1}=z_{1}$. Применяя лемму 8 , получаем нужное равенство.

ЛЕмма 10. Для любой приведенной матрицы $М$ второго вида из (2.1) выполняется неравенство

$$
R \leqslant \frac{1}{3} D(M)
$$

ДокАЗАтЕЛьство. Рассмотрим разложение $M$ по первому столбцу

$$
D(M)=x_{1}\left(y_{2} z_{3}+y_{3} z_{2}\right)+x_{2}\left(y_{1} z_{3}-y_{3} z_{1}\right)+x_{3}\left(y_{1} z_{2}+y_{2} z_{1}\right),
$$

в котором все три слагаемых не отрицательны. В случае $x_{1} \leqslant z_{1}$ нужное неравенство доказано в лемме 8.

Рассмотрим случай $x_{1}>z_{1}$. Если $X=R$, то по неравенству Коши

$$
\begin{aligned}
D(M) & \geqslant 5\left(x_{1} y_{2} z_{3} \cdot x_{1} y_{3} z_{2} \cdot x_{2} y_{1} z_{3} \cdot x_{3} y_{1} z_{2} \cdot x_{3} y_{2} z_{1}\right)^{1 / 5}-x_{2} y_{3} z_{1} \\
& =5(X Y Z)^{2 / 5}\left(x_{2} y_{3} z_{1}\right)^{-1 / 5}-x_{2} y_{3} z_{1} \\
& \geqslant 5(X Y Z)^{2 / 5} X^{-1 / 5}-X \geqslant 5 X^{6 / 5} X^{-1 / 5}-X=4 X .
\end{aligned}
$$

Это даже лучше, чем требуется.

Пусть теперь $X>R$. При уменьшении $x_{3}$ на отрезке $\left[y_{3}, z_{3}\right]$ отношение $R\left(x_{3}\right) / D\left(x_{3}\right)$ увеличивается до тех пор, пока не возникнет равенство $X=R$ или $x_{3}=y_{3}$. Как мы только что показали, в первом случае нужное неравенство вьполнено, а во втором оно следует из леммы 9. Лемма 10 полностью доказана.

ЗАмЕчАнИЕ 3 . Из доказательства видно, что в лемме 10 равенство возникает только для приведенных матриц вида

$$
\left(\begin{array}{ccc}
x_{1} & -2 x_{1} & -2 x_{1} \\
x_{2} & x_{2} / 2 & -x_{2} / 2 \\
x_{3} & x_{3} & x_{3}
\end{array}\right)
$$

с положительньми $x_{1}, x_{2}, x_{3}$.

Из лемм $6,7,10$ и замечания 3 непосредственно следует оценка (3.1), а вместе с ней и утверждение сформулированной во введении теоремы.

Постоянная $\frac{1}{3}$ в доказанной теореме не может быть заменена меньшей по следующей причине.

Пусть $a$ и $b$ целые, а $N$ - натуральное число. Рассмотрим решетки вида

$$
\Gamma_{N}(a, b)=\left\{\frac{1}{N^{1 / 3}}\left(m_{1}, m_{2}, N m_{3}+a m_{1}+b m_{2}\right): m_{1}, m_{2}, m_{3} \in \mathbb{Z}\right\} .
$$

По теореме Линника-Скубенко (см. [10]) любую приведенную матрицу с определителем 1 со сколь угодной точностью (в равномерной метрике) можно аппроксимировать матрищей, соответствующей некоторому базису решетки из $\Gamma_{N}(a, b)$. Поэтому наименњшая возможная константа из правой части теоремы совпадает с соответствующей константой для минимальных и приведенных матриц, равной $\frac{1}{3}$. 


\section{Зак лючение}

Пусть $P$ - растущий положительный параметр. Через $N_{\alpha, \beta}(P)$ обозначим количество знаменателей $q$ к $(\alpha, \beta)$, не превосходящих $P$. Есть основание считать, что справедлива

ГИПотЕЗА. Для некоторой абсолютной положительной константы $C$, не зависящей от $(\alpha, \beta)$,

$$
\lim _{P \rightarrow \infty} \frac{N_{\alpha, \beta}(P)}{\ln ^{2} P}=C
$$

при почти всех $(\alpha, \beta) \in(0,1)^{2}$ по мере Лебега.

Речь идет о обобщении асимптотической формулы Хинчина-Леви (см. [11]).

$$
\lim _{P \rightarrow \infty} \frac{N_{\alpha}(P)}{\ln P}=\frac{\pi^{2}}{12 \ln 2}
$$

для почти всех $\alpha \in(0,1)$. Здесь $N_{\alpha}(P)$ есть количество подходящих дробей $a_{i} / q_{i}$ к $\alpha$, для которых $q_{i} \leqslant P$.

Отметим, что при всех $(\alpha, \beta) \in(0,1)$ выполняется оценка $N_{\alpha, \beta}(P)=O\left(\ln ^{2} P\right)$ (В.А. Быковский, неопубликовано). С помощью технических приемов из работы [8] нетрудно показать, что если $1, \alpha, \beta$ рационально независимы, то любой знаменатель $q_{1}$ к $(\alpha, \beta)$ можно дополнить до невырожденной тройки $\left\{q_{1}, q_{2}, q_{3}\right\}$ смежных знаменателей к $(\alpha, \beta)$. Это наблюдение подчеркивает содержательность главного результата работы.

\section{Список литературы}

1. Vahlen K. Th. Über Nährungs-werthe und Kettenbrüch // J. Rein. Angew. Math. 1895. V. 115. №3. P. 221-233.

2. Венков Б. А. Элементарная теория чисел. М.: ОНТИ, 1937.

3. Хинчин А. Я. Цепные дроби. М.: Физматгиз, 1961.

4. Касселс Дж. Введение в теорию диофантовых приближений. М.: ИЛ, 1961.

5. Быковский В. А. Теорема Валена для двумерных подходящих дробей // Матем. заметки. 1999. Т. 66. № 1. С. 30-37.

6. Вороной Г. Ф. Собрание сочинений. Т. 1. Киев: Изд-во АН УССР, 1952.

7. Minkowski H. Generalisation de la theorie des fraction continues // Ann. Sci. École Norm. Sup. 1896. V. 13. № 2. P. 41.

8. Быковский В. А., Горкуша О.А. Минимальные базисы трехмерных полных решеток // Матем. сб. 2001. Т. 192. № 2. С. 57-66.

9. Горкуша О. А. Минимальные базисы трехмерных полных решеток // Матем. заметки. 2001. T. 69. № 3. C. 353-362.

10. Линник Ю. В., Скубенко Б. Ф. Асимптотическое распределение целочисленных матриц третьего порядка // Вестн. ЛГУ. Сер. матем., мех., астрон. 1964. Т. 3. № 13. С. 25-36.

11. Биллингслей П. Эргодическая теория и информация. М.: Мир, 1969.

Хабаровское отделение

Института прикладной математики ДВО РАН

Поступила в редакцию

E-mail: vab@iam.khv.ru, mariya@iam.khv.ru

17.06.2002 